\title{
Foot Pressure and Gait Analysis of Children's Shoes
}

\author{
Cong Li, Yanzhen Wang, Daomiao Zhang
}

Shanghai University of Engineering and Technology, School of Fashion, Shanghai 20000, China.

\begin{abstract}
Shoes are indispensable daily necessities in life. Good shoes not only prevent foot damage, but even adjust walking posture. A pair of comfortable children's shoes can prevent children's foot distortion and help them correct walking gait (purpose). Using the Trigno Lab wireless electromyography acquisition system produced by Delsys, USA, the gait EMG data was collected from different parts of the children during walking with different materials and hardness $(50,55,60)$. The SPSS software used the collected data. Analysis, to get the material and hardness (method) of children's shoes suitable for children. During the walking process, the muscle movement causes the myoelectric signal to change. The myoelectric signal is roughly in the front and back. The front part of the main muscle has slightly more than the back part. The shoes of different materials and hardness have slightly different electromyographic signals. (Results) Experimental analysis showed that when walking freely, the tibialis anterior muscle activity was greater at TPR60, and the gastrocnemius muscle activity was greater at TPR55, while the hardness had less effect on the biceps femoris and lateral femoral muscle. At the hardness of 55, the wearer's EMG signal of the rubber material sole is the largest, and the muscle activity is relatively large. For the gastrocnemius muscle, the muscle activity of the TPR material is greater, and the material has more influence on the biceps femoris and the lateral femoral muscle. Small (conclusion).
\end{abstract}

Keywords: Children; gait; wireless electromyography; myoelectric signals; data analysis.

\section{Introduction}

David Hayes, a famous British podiatrist, who designed Dordorhorse children's health shoes, found from his experience that most newborn babies' feet are healthy but $60 \%$ of adults are not. He pointed out that it is caused by improper shoes during the development of children's feet; in China, National public welfare project "research on china's population foot gauge by Qiu Li, etc., shows that the majority of the feet of Chinese kindergarten children are healthy, while the number of unhealthy feet from middle school students in the same area reach as high as 80 percentage. Secondly, the Chinese people have a high incidence of hallux valgus deformity and flat feet, especially in economically developed areas. All of the above researches indicate that there is a widespread problem of children wearing shoes at home and abroad. In a word, the design of children's shoes is not reasonable enough [1].

Limb movements [2] are the result of the interaction of nerves and muscles under the command of the brain. Surface electromyography (SEMG) is a weak non-stationary electrical signal accompanied by muscle contraction. It contains important neuromuscular information and is widely used in rehabilitation medicine, human-machine interface and other fields. Based on the mechanism of the generation of myoelectric signals, this experiment chooses the basic movements (walking) of the human body that can characterize the strength of muscle activity to make an electromyography test on children wearing different shoes (different hardness and material). through the qualitative analysis of the strength, weakness, and attenuation of the signal. We will try to find the data parameters of the children's shoes suitable for children. It can provide technical guidance for the production of the enterprises and some suggestions for the consumers.

\section{Research Object and Method}

\subsection{Laboratory Apparatus}

\subsubsection{Trigno Lab Full Wireless Surface EMG Test System}

This experiment uses the Trigno Lab full wireless surface electromyography test system produced by Delsys. It mainly consists of several testers, sensors, acquisition systems, software analysis 
systems, and desktop computers. These include 16 surface electromyography channels and 48 threedimensional accelerometer channels. The signal frequency is $2000 \mathrm{~Hz}$, the effective distance is $40 \mathrm{~m}$, and the EMG signal can be continuously collected for 8 hours. Data can be recorded simultaneously and monitored in real time, with no delay between the sensor and the acquisition system. The EMG works Analysis System performs a variety of signal processing and data analysis.
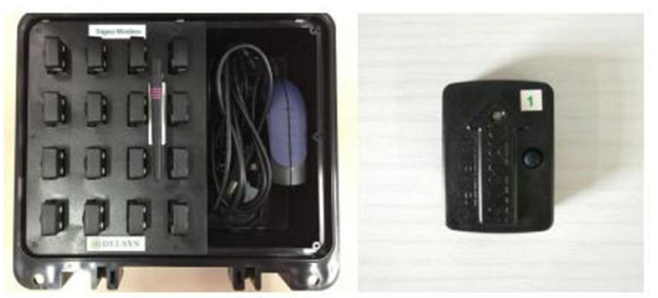

Fig. 1 Two or more references

\subsubsection{Experimental Site}

Leg movement is the result of the interaction between the nervous system, the muscles and joints of the legs under the control of the cerebral cortex [3], while the muscles in exercise are mainly quadriceps, which are used to stretch the knee joint, and the biceps femoris is mainly responsible for bending the knee. Stretch your hips. The tibialis anterior muscle prevents the soles of the feet from patping the ground while walking, and at the same time, stabilizes the ankle joint and assists in pushing the trunk forward. The medial head of the gastrocnemius and the lateral head of the gastrocnemius have both knee flexion and plantar flexion, which can provide power for walking. The test of myoelectric signals is the test of the electromechanical signals of these muscles.

Table 1. Basic information of the muscles

\begin{tabular}{cc}
\hline Tibialis Anterior & TA \\
Gastrocnemius Medial & GM \\
Gastrocnemius Lateral & GL \\
Biceps Femoris & BF \\
Vastus Lateralis & VL \\
\hline
\end{tabular}

Find 6 children (female) aged 12-13 years old, weighing 40kg-50kg, with height meeting 140$160 \mathrm{CM}$. Inform the testing process and precautions. Wipe the attachment point of the experimenter's sensor with an alcohol cotton ball to prevent dust, dander, and hair from affecting the test results. The No. 1 sensor was placed on the tibialis anterior muscle of the lower limb, the No. 2 sensor was placed on the medial side of the gastrocnemius muscle, the No. 3 sensor was placed on the lateral side of the gastrocnemius muscle, the No. 4 sensor was placed on the biceps femoris, and the No. 5 sensor was attached to the external muscle of the femoral artery. When attaching the sensor, note that the sensor number must correspond to the corresponding number of the muscle.

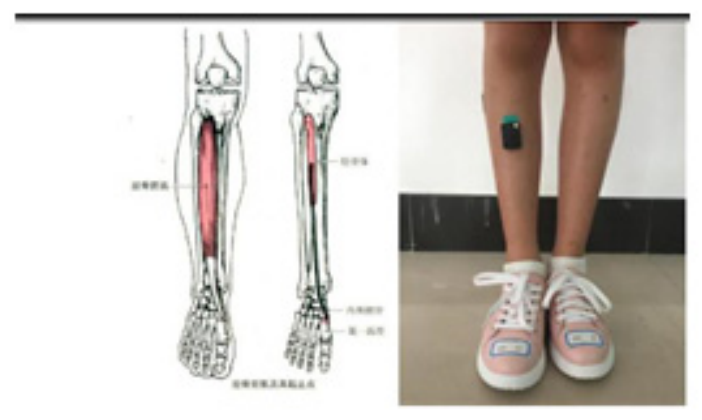

Fig.2 Anatomy of the sacrum muscles and sensor placement 


\subsection{Experimental Scheme}

The Shore A hardness tester measures the three pairs of experimental shoes designed by the author [4]. The hardness values of the experimental design are 50 degrees, 55 degrees, and 60 degrees respectively. The experimental shoes use a cross design method. When the sole hardness is 55 . When Shore A is used, it corresponds to three different sole materials of TPR, MD and rubber; when the sole material is TPR, it corresponds to 50 different Sole A, 55 Sower A and 60 Sower A. The schematic diagram of the experimental shoes is as follows.

Table 2. shoes with different hardness and different materials

\begin{tabular}{ccc}
\hline number & Sole Hardness (Shore A) & Sole material \\
1 & 50 & TPR \\
2 & 55 & RUBBER \\
3 & 55 & TPR \\
4 & 55 & MD \\
5 & 60 & TPR \\
\hline
\end{tabular}

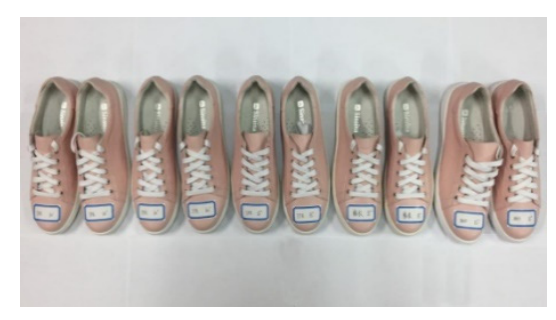

Fig. 3 shoes with different materials and hardness

Let the tester perform a test on the $10 \mathrm{~m}$ trail for a few minutes. If the spindle type EMG is obtained, the placement position is correct. If the messy EMG signal is obtained, the sensor position is incorrectly placed and needs to be reattached. In the constant temperature room, the temperature is $26^{\circ} \mathrm{C}$, the test distance is $10 \mathrm{~m}$, and the device is worn for $1-2$ minutes before the experiment to eliminate the discomfort when wearing the device. The testers walked in normal posture with shoes of different materials and hardness. The instructor also recorded the EMG signal, and each pair of shoes repeated experiments 5-6 times [4].

\subsection{Experimental Index}

Selection and description of indicators: 1 integral EMG [5] (IEMG) refers to the area of the EMG image per unit time, and can reflect the number of muscle fibers during exercise and the discharge size of each exercise unit. $2 \mathrm{rms}$ amplitude, refers to the root mean square value within a certain period of time, used to describe the average variation characteristics of myoelectricity. Used to estimate the amount of force generated. 3 Mean EMG (AEMG), an indicator of the amplitude change of the EMG signal, indicating the number of activations of the motor unit, related to central control.

Surface electromyography (SEMG) is a surface electromechanical device that guides and records a series of changes in neuromuscular signals from the surface of the skin, which can be used to reflect some information about neuromuscular activity. The intensity of the EMG signal and the magnitude of the amplitude can directly reflect the contraction and strength of the muscle. The denser the myoelectric signal, the greater the amplitude, the stronger the contraction. 


\section{Result}

\subsection{EMG Analysis}

As can be seen from the figure below, the muscle discharge has a periodic change. The medial and lateral discharge of the gastrocnemius muscle is discharged earlier and at the same time. The tibialis anterior muscle discharge is slightly delayed, and the biceps femoris and lateral femoral muscles are discharged almost at the same time. From the EMG signal response, we can conclude that during the walking process, the gastrocnemius muscle first moves, then the tibialis anterior muscle re-energizes, and the muscles in the thigh area finally exert force. It can be seen from the figure that the myoelectric signals of the tibialis anterior and gastrocnemius muscles are dense, indicating that the muscle movement of these parts is large, playing a major role in walking, and the signal intensity of the biceps femoris and lateral femoral muscles is small, indicating these muscles. It plays an auxiliary role in the movement process. The medial and lateral gastrocnemius had the longest discharge time, followed by the tibialis anterior muscle, and the lateral femoral muscle. The shortest discharge time was the biceps femoris. It is indicated that the muscle activity of the medial and lateral tibialis anterior muscle of the gastrocnemius muscle is higher during walking.

The effect of the hardness of the sole and the material on the myoelectric properties of the lower limb muscles during walking: 1 In the $10 \mathrm{~m}$ free walking experiment, due to the difference in material, hardness and experimenter of the children's shoes, Inconsistent use. Therefore, the average electromyogram (AEMG) is used as an indicator to indicate the average work size. It is equal to the ratio of the integral of the time series to the time, which is the size of the work per unit time, thus eliminating the effect of the time difference on our results. In order to reduce the error, we adopt a single individual analysis method, using a variety of experimental data, presenting by means of mean value plus standard deviation, testing the experimental data for homogeneity of variance, and analyzing the hardness of the sole to the EMG signal by single factor in SPSS. The impact [7].

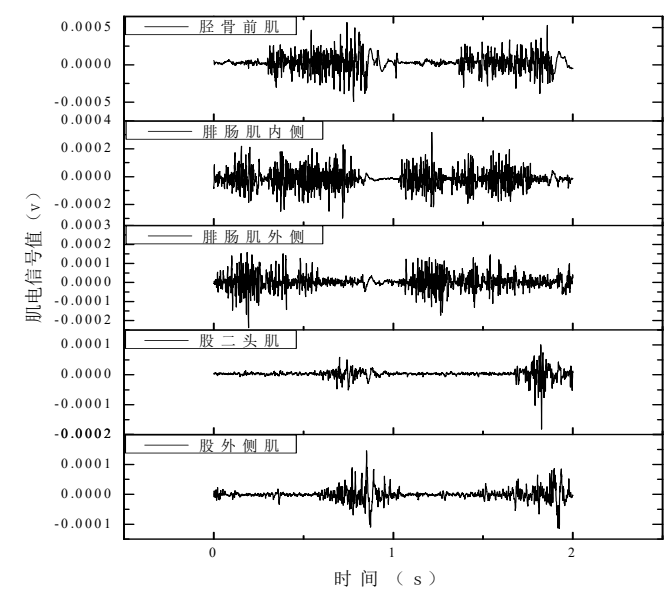

Fig. 4 Electromyogram

\subsection{The Effect of Shoes Hardness on Myoelectric Signal}

It can be seen from Table 3 that the material and hardness of the sole have certain influence on the EMG signal, but there are obvious differences between the individuals. The hardness of the sole of the third tester has little influence on the EMG signal, and the muscles of the lower limbs are The AEMG values have no significant effect and may be related to the subject's walking posture and physical state. The hardness of the sole has a significant effect on the AEMG value of the tibialis anterior muscle of each subject, but different subjects respond differently to the hardness of the sole. Tester 1 has three effects on the EMG signal. The tester 2 has a hardness difference between 50 and 60 . The tester 4 has a small difference between the hardness of 50 and 60 , which is different from the difference of 55. Big. Tester 5 has a small difference between hardness 50 and 55, which is quite different from 60. Tester 6 has a small difference in hardness 50 and 60, which is quite different from 
55. As the hardness of the sole increased, the AEMG values of the tibialis anterior muscle of each subject showed different trends. The trend of change between the subject 1 and the subject 2 is inconsistent, and the rest of the subjects are first reduced and then increased. The AEMG value of the tibialis anterior muscle of the subject was the largest, and the average tibialis anterior muscle had the highest work intensity during walking [8].

From the surface of the gastrocnemius muscle EMG signal can be seen, in the 1, 2, 4 testers at a hardness of 55 when the EMG signal is stronger, the No. 1 tester signal amplitude is the largest, reaching 9, 2, 4 although there are differences, but the difference is relatively small. On the 5th, the tester happened to be the opposite. At a hardness of 55, the myoelectric signal was the smallest and the overall difference was small. There was no significant difference in No. 3 myoelectricity. When the hardness of No. 6 was 55, the EMG signal was weak. The electromyographic signals of different wearers also have obvious differences. The myoelectric signals of No.1 and No.6 are much larger than those of No.2,3,4,5, and the difference of EMG signals of 2,3,4,5 Not big. For the lateral myoelectric signal of the gastrocnemius, the electromyographic signals of 1,2 , and 4 are greater when the hardness of the shoe is 55, while the myoelectric signal of the 6th is the smallest, and the influence of the hardness of the shoe on the electromyographic signal of the $3 \mathrm{rd}$ is not It is clear. Tester No. 5 was the weakest EMG signal at 60 Shore. The effects of individual differences on myoelectric signals are quite different.

Table 3. EMG signals of shoes with different hardness

\begin{tabular}{ccccccc}
\hline number hardness & TA & GM & GL & BF & VL \\
\hline \multirow{3}{*}{1} & TPR50 & $46.22 \pm 1.63 \mathrm{ab}$ & $25.67 \pm 1.62 \mathrm{ab}$ & $17.11 \pm 1.05 \mathrm{a}$ & $8.13 \pm 0.68$ & $6.49 \pm 0.61 \mathrm{ab}$ \\
& TPR55 & $67.27 \pm 0.51 \mathrm{ac}$ & $34.92 \pm 2.69 \mathrm{ac}$ & $20.63 \pm 1.81 \mathrm{ab}$ & $7.77 \pm 0.75$ & $7.21 \pm 0.64 \mathrm{ac}$ \\
& TPR60 & $60.26 \pm 2.30 \mathrm{bc}$ & $27.96 \pm 2.83 \mathrm{bc}$ & $18.47 \pm 1.61 \mathrm{~b}$ & $8.36 \pm 0.72$ & $5.43 \pm 0.40 \mathrm{bc}$ \\
\multirow{3}{*}{2} & TPR50 & $31.14 \pm 0.90 \mathrm{c}$ & $21.14 \pm 0.93 \mathrm{a}$ & $9.18 \pm 1.12 \mathrm{a}$ & $15.13 \pm 1.87$ & $5.22 \pm 0.54$ \\
& TPR55 & $32.19 \pm 1.30$ & $22.69 \pm 0.85 \mathrm{ab}$ & $11.66 \pm 1.05 \mathrm{ab}$ & $16.83 \pm 1.93$ & $5.13 \pm 0.85$ \\
& TPR60 & $32.25 \pm 0.72 \mathrm{c}$ & $21.58 \pm 0.56 \mathrm{~b}$ & $9.76 \pm 0.61 \mathrm{~b}$ & $14.83 \pm 1.36$ & $5.13 \pm 0.57$ \\
\multirow{3}{*}{3} & TPR50 & $32.79 \pm 0.92$ & $21.49 \pm 0.68$ & $11.77 \pm 0.21$ & $9.05 \pm 0.78$ & $9.11 \pm 0.67$ \\
& TPR55 & $32.45 \pm 1.05$ & $21.41 \pm 0.44$ & $11.02 \pm 0.99$ & $9.04 \pm 0.99$ & $8.59 \pm 1.36$ \\
& TPR60 & $33.02 \pm 3.12$ & $21.05 \pm 2.56$ & $11.38 \pm 1.48$ & $9.43 \pm 0.91$ & $9.46 \pm 1.28$ \\
4 & TPR50 & $36.67 \pm 2.06 \mathrm{ac}$ & $19.59 \pm 0.60 \mathrm{a}$ & $11.74 \pm 1.89 \mathrm{a}$ & $11.02 \pm 1.15$ & $11.24 \pm 2.35$ \\
& TPR55 & $31.93 \pm 2.42 \mathrm{a}$ & $20.37 \pm 0.68 \mathrm{ab}$ & $14.80 \pm 1.92 \mathrm{ab}$ & $8.21 \pm 0.49$ & $10.36 \pm 1.14$ \\
& TPR60 & $33.30 \pm 1.52 \mathrm{c}$ & $19.14 \pm 0.43 \mathrm{~b}$ & $11.17 \pm 0.97 \mathrm{~b}$ & $6.99 \pm 0.35$ & $12.71 \pm 1.14$ \\
\multirow{2}{*}{5} & TPR50 & $32.46 \pm 0.93$ & $23.39 \pm 1.42$ & $15.61 \pm 1.18$ & $11.54 \pm 1.08$ & $5.92 \pm 0.47 \mathrm{ac}$ \\
& TPR55 & $31.84 \pm 0.52 \mathrm{~b}$ & $22.80 \pm 0.59$ & $15.61 \pm 1.42$ & $12.08 \pm 0.90$ & $16.35 \pm 0.74 \mathrm{ab}$ \\
& TPR60 & $33.02 \pm 3.12$ & $21.05 \pm 2.56$ & $11.38 \pm 1.48$ & $9.43 \pm 0.91$ & $9.46 \pm 1.28$ \\
& TPR50 & $37.55 \pm 1.83 \mathrm{a}$ & $45.84 \pm 2.53$ & $11.42 \pm 0.76$ & $12.98 \pm 1.26 \mathrm{ac}$ & $4.62 \pm 1.01 \mathrm{c}$ \\
& TPR55 & $35.65 \pm 0.52 \mathrm{ab}$ & $47.96 \pm 2.81$ & $9.84 \pm 0.38$ & $10.89 \pm 0.77 \mathrm{a}$ & $4.30 \pm 0.55 \mathrm{~b}$ \\
& TPR60 & $37.22 \pm 1.25 \mathrm{~b}$ & $47.42 \pm 2.10$ & $14.79 \pm 0.33$ & $11.71 \pm 0.50 \mathrm{c}$ & $5.66 \pm 0.69 \mathrm{bc}$ \\
\hline
\end{tabular}

From the size of the biceps muscle EMG signal in Table 3, it can be seen that the myoelectric signal is stronger when the hardness is 55 Shore. On the contrary, 1, 4, and 6 are weaker at 55 Shore. There was no significant difference in the No. 3 myoelectric signal, indicating that the hardness had no significant effect on the No. 3 myoelectric signal. The difference between different individuals is relatively large, the No. 1 EMG signal is the weakest, and the No. 2 EMG signal is the strongest [9].

At the same time, we can see the signal of the lateral femoral muscle. The amplitude of the 2, 3, and 4 EMG signals is not very large. On the 1 st and 5th, the myoelectric signal was the strongest at hardness 55, and the difference in hardness on the 5th had a significant effect on the myoelectric 
signal. On the 6th, the EMG signal was not much different at the hardness of 50 and 55 Shore, and the EMG signal was strong at 60 Shore.

\subsection{Effect of Materials on Myoelectric Signals}

It can be seen from Table 4 that different individuals have significant effects on the EMG signals in the biceps and lateral femoral muscles. It can be seen from the table that the myoelectric signal is more affected by the shoe material, and the individual difference is relatively large. We present the myoelectric signals of different materials, different muscles, and different individuals in a histogram.

Table 4. Electromyographic signals of shoes with different hardness of 55

\begin{tabular}{ccccccc}
\hline number & Shoe type & TA & GM & GL & BF & VL \\
001 & TPR55 & $48.21 \pm 1.79$ & $25.63 \pm 1.31$ & $15.16 \pm 1.28$ & $5.7 \pm 0.48$ & $5.3 \pm 0.4 \mathrm{f}$ \\
& rubber 55 & $46.99 \pm 2.25$ & $25.37 \pm 2.08$ & $15.15 \pm 0.81$ & $6.63 \pm 0.55$ & $5.87 \pm 0.44$ \\
& MD55 & $46.3 \pm 2.26$ & $25.14 \pm 2.72$ & $14.6 \pm 0.62$ & $7.23 \pm 3.33$ & $6.73 \pm 1.57 \mathrm{f}$ \\
002 & TPR55 & $36.4 \pm 0.92$ & $25.67 \pm 0.90$ & $13.16 \pm 0.61$ & $19.00 \pm 1.76$ & $5.78 \pm 0.72 \mathrm{~d}$ \\
& rubber 55 & $37.24 \pm 1.75$ & $24.7 \pm 0.99$ & $12.41 \pm 0.93$ & $18.41 \pm 2.53$ & $7.24 \pm 0.69 \mathrm{de}$ \\
& MD55 & $36.41 \pm 0.88$ & $25.64 \pm 0.91$ & $13.13 \pm 0.61$ & $19.03 \pm 1.75$ & $5.79 \pm 0.71 \mathrm{e}$ \\
003 & TPR55 & $39.35 \pm 0.99 \mathrm{~d}$ & $26.00 \pm 1.30 \mathrm{f}$ & $13.34 \pm 0.78 \mathrm{df}$ & $10.94 \pm 0.88$ & $10.37 \pm 1.2$ \\
& rubber 55 & $40.44 \pm 0.72 \mathrm{~d}$ & $25.49 \pm 0.55 \mathrm{e}$ & $12.18 \pm 0.38 \mathrm{~d}$ & $11.62 \pm 0.88$ & $10.27 \pm 0.98$ \\
& MD55 & $40.33 \pm 0.77$ & $23.77 \pm 1.16 \mathrm{ef}$ & $12.47 \pm 0.41 \mathrm{f}$ & $11.79 \pm 0.33$ & $11.63 \pm 1.76$ \\
004 & TPR55 & $35.51 \pm 2.71 \mathrm{~d}$ & $22.76 \pm 2.43$ & $16.35 \pm 0.63$ & $12.16 \pm 6.68$ & $13.22 \pm 3.60$ \\
& rubber 55 & $38.73 \pm 3.09 \mathrm{~d}$ & $21.67 \pm 0.74$ & $14.58 \pm 1.14$ & $11.36 \pm 2.04$ & $13.65 \pm 1.76$ \\
& MD55 & $37.44 \pm 1.03$ & $22.77 \pm 0.87$ & $14.63 \pm 0.43$ & $11.77 \pm 0.92$ & $13.39 \pm 0.5$ \\
& TPR55 & $32.38 \pm 1.47 \mathrm{~d}$ & $23.19 \pm 1.05 \mathrm{df}$ & $15.84 \pm 1.06 \mathrm{~d}$ & $12.27 \pm 0.83 \mathrm{df}$ & $16.33 \pm 1.98 \mathrm{~d}$ \\
& ubber55 & $36.67 \pm 1.92 \mathrm{de}$ & $24.94 \pm 0.91 \mathrm{~d}$ & $13.18 \pm 1.36 \mathrm{de}$ & $13.76 \pm 1.38 \mathrm{de}$ & $11.45 \pm 1.17 \mathrm{de}$ \\
& MD55 & $33.21 \pm 2.08 \mathrm{e}$ & $24.59 \pm 0.97 \mathrm{f}$ & $15.01 \pm 1.65 \mathrm{e}$ & $10.7 \pm 0.81 \mathrm{ef}$ & $16.5 \pm 3.19 \mathrm{e}$ \\
& TPR55 & $32.83 \pm 0.56$ & $44.12 \pm 1.56 \mathrm{df}$ & $9.06 \pm 0.5 \mathrm{df}$ & $10.03 \pm 0.74 \mathrm{~d}$ & $3.96 \pm 0.51 \mathrm{~d}$ \\
& rubber 55 & $31.06 \pm 2.13 \mathrm{e}$ & $39.33 \pm 2.34 \mathrm{~d}$ & $12.35 \pm 1.04 \mathrm{de}$ & $11.86 \pm 0.77 \mathrm{de}$ & $5.4 \pm 0.55 \mathrm{de}$ \\
& MD55 & $34.57 \pm 1.46 \mathrm{e}$ & $40.8 \pm 2.23 \mathrm{f}$ & $10.32 \pm 0.51 \mathrm{ef}$ & $10.44 \pm 0.48 \mathrm{e}$ & $3.87 \pm 0.48 \mathrm{e}$ \\
\hline
\end{tabular}

It can be seen from the table that the difference between TPR material and rubber material in 3, 4, and 5 is significant, and 5 and 6 are the difference between rubber and MD material. The muscle activity of the three, 1, 2, and 4 bodies decreased in turn. The No. 3 individual myoelectric signal was relatively stable, indicating that the material had the least effect on the muscle activity of No. 3 . It can be seen from the table that the difference between TPR material and rubber material in 3, 4, and 5 is significant, and 5 and 6 are the difference between rubber and MD material. The muscle activity of the three, 1, 2, and 4 bodies decreased in turn. The No. 3 individual myoelectric signal was relatively stable, indicating that the material had the least effect on the muscle activity of No. 3 [10].

As can be seen from the table, different materials have less effect on the myoelectricity of the medial gastrocnemius. There were differences in the individual's myoelectric signals. There were no significant differences in 1, 2, 3, and 5, 4 was relatively low, and the difference in No. 6 was very obvious, indicating that the individual differences were relatively large, but the differences in materials were relatively small on the 6th. The muscle activity of the extrafusal muscles is small, and the myoelectric signals between individuals are also significantly different. No. 5 and No. 6 have obvious differences between rubber and MD materials, and there is also a significant difference between No. 5 TRP and rubber. 
There are significant differences in the myoelectric signals between the biceps when walking, and the activity of the biceps 2 and 1 is the most obvious and least obvious. 4, 6 in the TPR and rubber materials, the difference in myoelectric signals is large, 5,6 in the rubber and MD materials, the difference in myoelectric signals. As can be seen from the data of the lateral femoral muscle, there is a very large difference in muscle activity between individuals, between 1,2 , and 6 .

The difference is small, and the difference between 4 and 5 is also small. 2, 5, 6In the material is a large difference between rubber and MD, 2,6 rubber when the EMG signal is larger than MD, and 5 is the opposite, MD's EMG signal is stronger. 1,5,4 have significant differences in myoelectric signals in TPR and MD materials, 1,5 muscle activity is stronger in MD materials, and 4 muscle activity is stronger in materials with TPR.

\section{Summary}

By changing the myoelectric signal of 6 children wearing different materials and different hardness of the children's shoes when walking, we can see that, during free walking, the tibialis anterior muscle activity is greater at TPR60, and the gastrocnemius muscle activity is greater at TPR55. Hardness has less effect on the biceps femoris and lateral femoral muscles. At the hardness of 55, the wearer's EMG signal of the rubber material sole is the largest, and the muscle activity is relatively large. For the gastrocnemius muscle, the muscle activity of the TPR material is greater, and the material has more influence on the biceps femoris and the lateral femoral muscle. small. The myoelectric signal of the leg changes periodically. Among them, the tibialis anterior muscle and the gastrocnemius muscle have strong electromyographic signals, and the activity of the biceps femoris and lateral muscles is relatively weak. The hardness of the sole has the most significant effect on the AEMG value of the tibialis anterior muscle, and the AEMG value of the tibialis anterior muscle is the largest, and the effect on the medial length of the gastrocnemius muscle, the lateral gastrocnemius muscle and the lateral femoral muscle AEMG is significant, and the AEMG value of the biceps femoris is the least affected. Significant. The sole material has a significant effect on the AEMG values of the medial and lateral femoral muscles, followed by the medial and biceps muscles, with little effect on the tibialis anterior muscle. It can be seen that as the hardness of the sole increases, there is no uniform trend in the magnitude of AEMG values in the muscles of the lower limbs. The hardness of the sole has a significant effect on the activity of the tibialis anterior and gastrocnemius muscles, followed by the medial and lateral femoral muscles. The sole material has a greater influence on the activity of the tibialis anterior and lateral femoral muscles, followed by the medial and lateral of the gastrocnemius. Overall, the tibialis anterior muscle has the greatest contribution.

Changes in sole hardness and material have an effect on the pressure and gait of the child while walking. The specific conclusions are as follows:

Surface electromyography experiments showed that the changes in sole hardness and material had an effect on the activity of lower limb muscles during free walking, but there was no uniform trend in the changes of muscles.

Outlook: Due to the limited time and energy of individuals and the limitations of scientific research, there are many areas for improvement. Surface electromyography can be combined with kinematics to study; use modeling method to simulate human walking process on computer, simulate repeated test; use subjective evaluation scale, combined with physiology (electromyography, blood flow oxygen, etc.), Psychology (heart rate) conducts a comprehensive study of the exercise process.

\section{Suggest:}

In addition to the size of the shoe size, the hardness of the children's shoes manufacturer, the material, but also to indicate its efficacy and role, suitable for the crowd. Parents need to choose the right shoes according to the actual situation of their children. If necessary, take the children to the hospital for examination. Follow the doctor's advice to choose shoes to prevent the deformity of the children's feet.

insufficient: 
1) In this paper, the hardness of the sole is small, and several sets of different sole hardnesses can be set up for further study.

2) The number of experimental personnel also needs to be increased to reduce the error and improve the accuracy of the experimental data.

\section{Acknowledgments}

Shanghai University of Engineering and Technology Innovation Project (E3-0903-17-01110); Apparel Structure Design (K201709001). Clothing structure design (1) (A1-0601-18-01072).

\section{References}

[1]. ZHANG Xiuli, ZHU Xiaolan. Biomechanical characteristics of children's foot and design of children's shoes[J]. Journal of Beijing Sport University,2010,33(06):57-61.

[2]. Cao Qingqing. Research on limb movement recognition for multi-modal interpersonal interaction [D]. Harbin Institute of Technology, 2012.

[3]. Yin Xiaolin. Biomechanical analysis of the movement of Sanda whip leg [D]. Shanghai Institute of Physical Education, 2013.

[4]. Fang Dongdong. Effect of calf strap on the surface EMG signal of thigh in whole body vibration training [D]. Shanghai Institute of Physical Education, 2017.

[5]. GUO Jianlong. Study on the variation characteristics of surface integral EMG and its average power frequency value of lower limb muscles during deep jumping[J]. Journal of Shandong Physical Education Institute,2007(06):68-71.

[6]. Song Yawei, Cai Wei, Pei Hengjing, Hua Yufei, Sun Wen. Changes in sole hardness and foot pressure in human walking [J]. Chinese Journal of Tissue Engineering Research and Clinical Rehabilitation, 2009, 13(46): 9113-9116.

[7]. Wu Xinyan, Chen Yuejun. Study on the Characteristics of Leg Muscle Electromyography in the Single-step Slip Cycle of Elite Short-distance Women's Speed Skaters[J].Journal of Capital College of Physical Education,2015,27(06):563-568.

[8]. Wang Guangxu. Research on pattern recognition of lower limbs based on surface EMG signals [D]. Hebei University of Technology, 2014.

[9]. Zhang Ruihong, Wang Rencheng, Jin Dewen, Zhang Jichuan. Detection and analysis of human myoelectric signal on lower limb surface[J]. Journal of Tsinghua University(Science and Technology), 2000(08): 73-76.

[10]. Zuo Jing. Research on fall recognition of surface EMG signal and plantar pressure [D]. Hangzhou University of Electronic Science and Technology, 2015. 